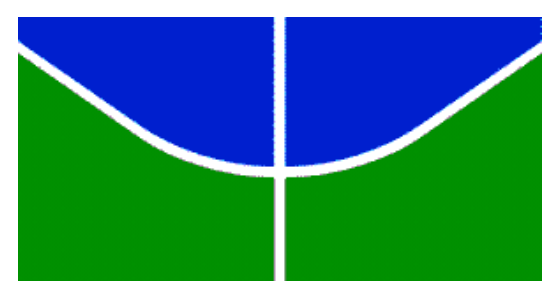

Universidade de Brasília-UnB

Instituto de Ciências Biológicas - IB

Programa de Pós-Graduação em Zoologia

\title{
Estudo comparativo sobre a dieta do Galbula ruficauda (Aves, Galbulidae) no Brasil central
}

\author{
Vitor César de Campos
}

Dissertação apresentada ao Programa de PósGraduação em Zoologia do Instituto de Ciências Biológicas, como requisito parcial para a obtenção do título de Mestre em Zoologia.

Orientador:

Dr. Carlos Eduardo Guimarães Pinheiro

Brasília, 2016 


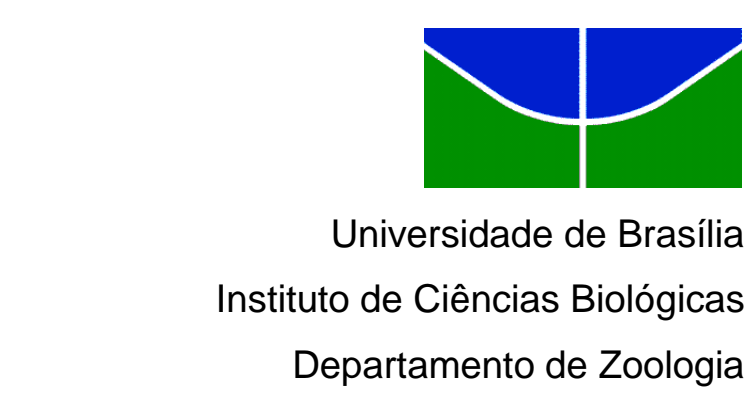

Programa de Pós Graduação em Zoologia

Dissertação de Mestrado

Vitor César de Campos

Titulo:

"Estudo comparativo sobre a dieta do Galbula ruficauda (Aves, Galbulidae) no Brasil central."

Banca Examinadora:

Prof. Dr. Carlos Eduardo Guimarães Pinheiro

Presidente/ orientador

Zoo/UnB

Prof. Dr. Reuber Albuquerque Brandão

Membro Titular

EFL/Unb
Prof. Dr. Raimundo Paulo Barros Henriques

Membro titular

$E C L /$ Unb

Prof. Dr. Ricardo Bomfim Machado

Membro suplente

Zoo/UnB

Brasília, 17 de março de 2016 


\section{AGRADECIMENTOS}

Agradeço aos meus pais, familiares, amigos e meu orientador pelo apoio, incentivo, ajuda e paciência que mostraram durante este período repleto de momentos bons e difíceis e também à natureza que nos fornece recursos, ainda que muitas vezes sem nos darmos conta, e proporciona espetáculos e curiosidades que tornam a Biologia mais do que um estudo ou uma profissão, mas sim um estilo de vida e uma paixão. 


\section{SUMÁRIO}

AGRADECIMENTOS __ iii

SUMÁRIO__ iv

ÍNDICE DE TABELAS___

ÍNDICE DE FIGURAS__ vi

RESUMO _

ABSTRACT_ viii

INTRODUÇÃO _ 1

Objetivos _ 3

MATERIAIS E MÉTODOS __ 3

Áreas de estudo __ 3

Métodos de campo _ 7

Aves investigadas no campo__ 8

Análise de variações na dieta e testes estatísticos empregados___ 9

RESULTADOS 9

DISCUSSÃO__ 17

CONCLUSÃO__ 18

REFERÊNCIAS BIBLIOGRÁFICAS _ 20 


\section{ÍNDICE DE TABELAS}

Tabela 1:As ordens de insetos e total de indivíduos capturados pelo Galbula ruficauda neste e outros estudos realizados no Brasil central e na Costa Rica. Dados relativos a sexo e estações do ano deste estudo foram agrupados.

Tabela 2: Resultados dos testes G (com correção de Willians) nas comparações realizadas entre sexo, estações do ano (Estação seca e Estação chuvosa) e áreas de estudo. Todos os testes com 2 graus de liberdade. 


\section{ÍNDICE DE FIGURAS}

Figura 1: Galbula ruficauda fêmea (esquerda) e macho (direita) (Fonte: Arquivo Pessoal).

Figura 2 - Visão geral da vegetação encontrada na Área 1 (Fonte: Arquivo Pessoal)

Figura 3 - Visão geral do trecho do Rio Maranhão encontrado na Área 1 (Fonte: Arquivo Pessoal)

Figura 4 - Visão geral da vegetação e do afluente do Rio Maranhão encontrados na Área 2 (Fonte: Arquivo Pessoal)

Figura 5 - Mapa com as localizações das áreas de estudo (Fonte: Google Earth Pro)

Figura 6 - Proporção (\%) de diferentes ordens de insetos na dieta de machos e fêmeas de Galbula ruficauda nas estações seca e chuvosa, com as Áreas de estudo ( 1 e 2 ) agrupadas.

Figura 7 - Proporção (\%) de indivíduos capturados no substrato (ou decolando) e em voo em cada uma das ordens de insetos consumidas por Galbula ruficauda.

Figura 8 - Proporção (\%) de Insetos Pequenos (= IP: Miscelânea e Díptera), Insetos Intermediários (= II: Hymenoptera) e Insetos Grandes (= IG: Coleoptera, Orthoptera, Odonata, Mantodea, Lepidoptera, e não identificados) na dieta do Galbula ruficauda separados por sexo, áreas de estudo e estações do ano. 


\section{RESUMO}

Este estudo compara a dieta do Galbula ruficauda entre sexos, estações do ano (estação seca e estação chuvosa) e dois locais relativamente próximos entre si ao longo da floresta de galeria do Rio Maranhão e de um de seus afluentes. Outras comparações envolvendo os resultados deste estudo com dados da dieta desta ave em outra área próxima as áreas de estudo deste projeto e uma área na América Central foram também realizados. Insetos atacados e consumidos por esta ave no Brasil central pertencem a sete ordens, incluindo: Hymenoptera, Diptera, Lepidoptera, Odonata, Coleoptera, Orthoptera e Mantodea. Insetos pequenos como Diptera e micro-Hymenoptera constituíram os itens mais abundantes na dieta desta ave, seguidos de Hymenoptera (especialmente vespas), Lepidoptera, Odonata, Coleoptera, Orthoptera e Mantodea. Comparações envolvendo a proporção destes itens na dieta destas aves não mostraram diferenças significativas entre sexos, mas mostraram fortes diferenças significativas entre as estações do ano. Comparações envolvendo diferentes locais no Brasil central mostraram diferenças significativas em alguns casos, mas não em outros. Comparações envolvendo locais no Brasil central e dados obtidos na Costa Rica também apresentaram fortes diferenças significativas entre eles.

Palavras-chave: ariramba, Brasil central, cerrado, dieta, estações do ano, Galbula ruficauda. 


\section{ABSTRACT}

This study compares the diet of the Galbula ruficauda between sexes, seasons (rainy season and dry season) and two locations relatively close to each other along a gallery forest of the Maranhão River and one of its tributaries in central Brazil. Additional comparisons involving the results obtained in this study with data on the diet of this bird in another site in Central Brazil and a forest site in Costa Rica were also carried out. Insects attacked and consumed by wild birds in central Brazil belong to seven orders including: Hymenoptera, Diptera, Lepidoptera, Odonata, Coleoptera, Orthoptera e Mantodea. Small insects like Diptera and microHymenoptera constituted the most abundant items in the diet of this bird, followed by relatively large Hymenoptera (especially wasps), Lepidoptera, Odonata, Coleoptera, Orthoptera and Mantodea. Comparisons involving the proportion of these items in the birds' diet showed no significant differences between sexes, but showed strong differences between seasons. Comparisons involving different locations in Central Brazil showed significant differences in some cases but not in others. Comparisons involving locations in central Brazil and data obtained in Costa Rica also furnished strong significant differences between them.

Key-words: central Brazil, cerrado, diet, Galbula ruficauda, Rufous-tailed jacamar, seasons. 


\section{INTRODUÇÃO}

O estudo da dieta de aves é um dos pilares para a compreensão de sua ecologia e seus comportamentos, uma vez que a forma como o alimento é obtido e os padrões encontrados em sua utilização determinam o sucesso relativo das espécies locais que estão em constante competição e permitem identificar fatores que determinam a organização da comunidade e estrutura das guildas (Scott 2013). Apesar disso, ainda são escassas informações de como a dieta de uma ave insetívora podem variar em diferentes biomas, como, por exemplo, a floresta chuvosa equatorial e o cerrado.

A espécie Galbula ruficauda (Cuvier 1816), objeto deste estudo e popularmente conhecida como ariramba-de-cauda-ruiva ou bico-de-agulha-de-rabovermelho, é uma ave neotropical, muito confundida com beija-flores por possuir um longo bico fino e pontudo, coloração verde-dourada na região dorsal e no peito, ferrugínea no abdômen e na parte de dentro da cauda. O macho apresenta uma faixa branca na garganta enquanto, na fêmea, essa região é parda (Gwynne et al. 2010) (Figura 1).

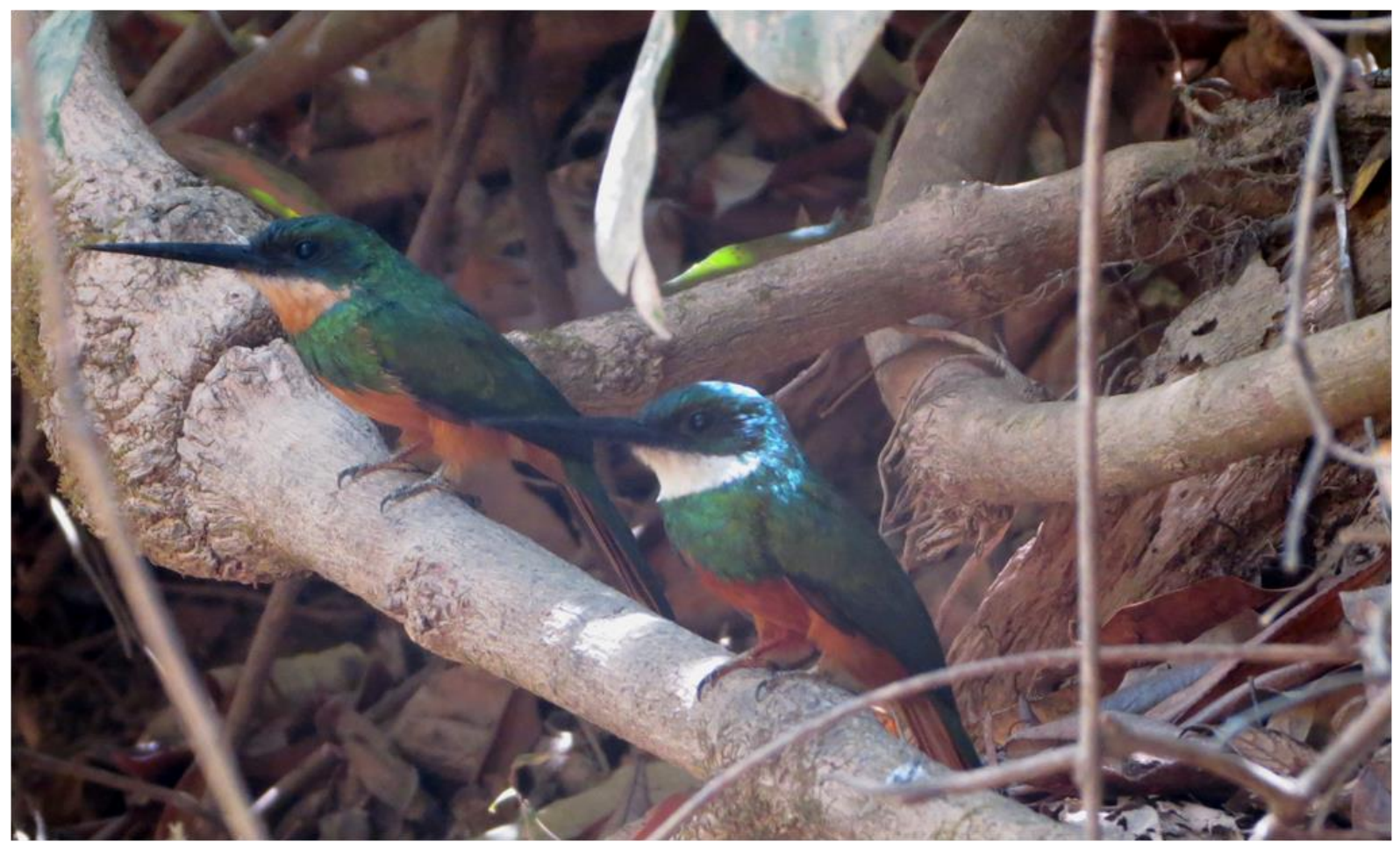

Figura 1 - Galbula ruficauda fêmea (esquerda) e macho (direita) (Fonte: Arquivo Pessoal). 
Adultos podem ser solitários, mas geralmente estão em pares (casais) ou em grupos de até cinco indivíduos. Constroem ninhos em barrancos argilosos ou arenosos, especialmente ao longo de rios e riachos e em cupinzeiros terrícolas (Sick 1997; Vasconcelos et al. 2015), sendo que o macho leva alimento para sua parceira quando ela está chocando. Os filhotes nascem com uma penugem esbranquiçada, mas em pouco menos de um mês adquirirem a coloração dos pais, podendo ser diferenciados pelo comprimento do bico, que é mais curto (Sick 1997).

A espécie G. ruficauda possui ampla distribuição geográfica, ocorrendo em florestas primárias e secundárias na América Central, na Amazônia e partes das regiões oeste, sudeste e centro sul do Brasil, Bolívia e nordeste da Argentina. No Brasil central está representado pela subespécie G. ruficauda rufoviridis (Cabanis 1851) que ocorre principalmente em matas de galerias de pequenos, médios e grandes rios desta região.

Sua dieta inclui uma grande variedade de insetos como Hymenoptera, Odonata, Lepidoptera, Diptera, Orthoptera e outros que podem ser capturados no ar, na vegetação ou sobre o chão (Chai 1986, Pinheiro et al. 2004), sendo que a grande maioria das informações sobre esta dieta consiste de relatos breves e observações ocasionais no campo (Skutch 1937, 1963; Moojen et al. 1941; Berla 1944; Slud 1964; Schubart et al. 1965; Sherry 1983; Novaes \& Lima 1998), ou dados de conteúdo estomacal (Poulin et al. 1994).

Estudos mais detalhados foram realizados por Fry (1970a, 1970b) no oeste brasileiro (Estado do Mato Grosso), que identificou uma variedade de espécies de Hymenoptera na dieta do Galbula ruficauda heterogyna (Todd 1932) e chegou a propor a hipótese de que o longo bico do G. ruficauda é uma adaptação primária para se alimentar destes insetos, mantendo os ferrões distantes da língua e outras partes sensíveis da boca.

Apesar de todos estes avanços, ainda não conhecemos com precisão como a composição da dieta de indivíduos adultos varia entre: 
1) diferentes regiões biogeográficas, como no caso da Floresta Tropical Úmida da Costa Rica, onde há uma estação seca de dezembro a março (Kernan \& Fowler 1995) e o Bioma Cerrado do Brasil central;

2) sexos, sendo que o único estudo já realizado com esta ave não encontrou diferenças significativas entre sexos (Pinheiro et al. 2004);

3) estações do ano, que apesar da marcante diferença climática e da composição de espécies de insetos presentes na estação seca e chuvosa do Brasil central, permanecem não investigadas;

4) locais relativamente próximos entre si, com semelhanças e diferenças na fisionomia da vegetação e na composição de espécies de insetos em geral.

\section{Objetivos}

1) realizar um estudo comparativo sobre a dieta do Galbula ruficauda entre sexos, locais e estações do ano, na região do Brasil central.

2) realizar um estudo comparativo da dieta do Galbula ruficauda entre diferentes biomas neotropicais (América Central vs Brasil central)

\section{MATERIAL E MÉTODOS}

\section{Áreas de estudo}

O local escolhido para o estudo foi o núcleo rural do Assentamento Contagem, localizado na região conhecida como FERCAL, Distrito Federal e as observações foram realizadas em matas de galeria presentes em duas propriedades particulares, com permissão dos proprietários.

A primeira, denominada Área 1 (Figura 2 e 3), se encontra junto à margem esquerda do Rio Maranhão (15²9'32.57"S, 4750'3.28"W) com área de floresta preservada de aproximadamente $6.579 \mathrm{~m}^{2}$. A segunda, denominada Área 2 (Figura 4), compreende a mata de galeria de um pequeno afluente do Rio Maranhão (15²9'9.66"S, 4751'18.77"W) com área aproximada de $12.852 \mathrm{~m}^{2}$ e distante $2 \mathrm{~km}$ 
da Área 1. Os dados obtidos no estudo realizado por Pinheiro et al. (2004) em uma área próxima aos locais amostrados no presente estudo (cerca de 1,6 Km da Área 1 e 3,6 Km da Área 2 (Figura 5)), e o de Chai (1986), utilizando o Galbula ruficauda melanogenia (Sclater, 1852) na Costa Rica, serão utilizados em comparações com os obtidos neste trabalho.

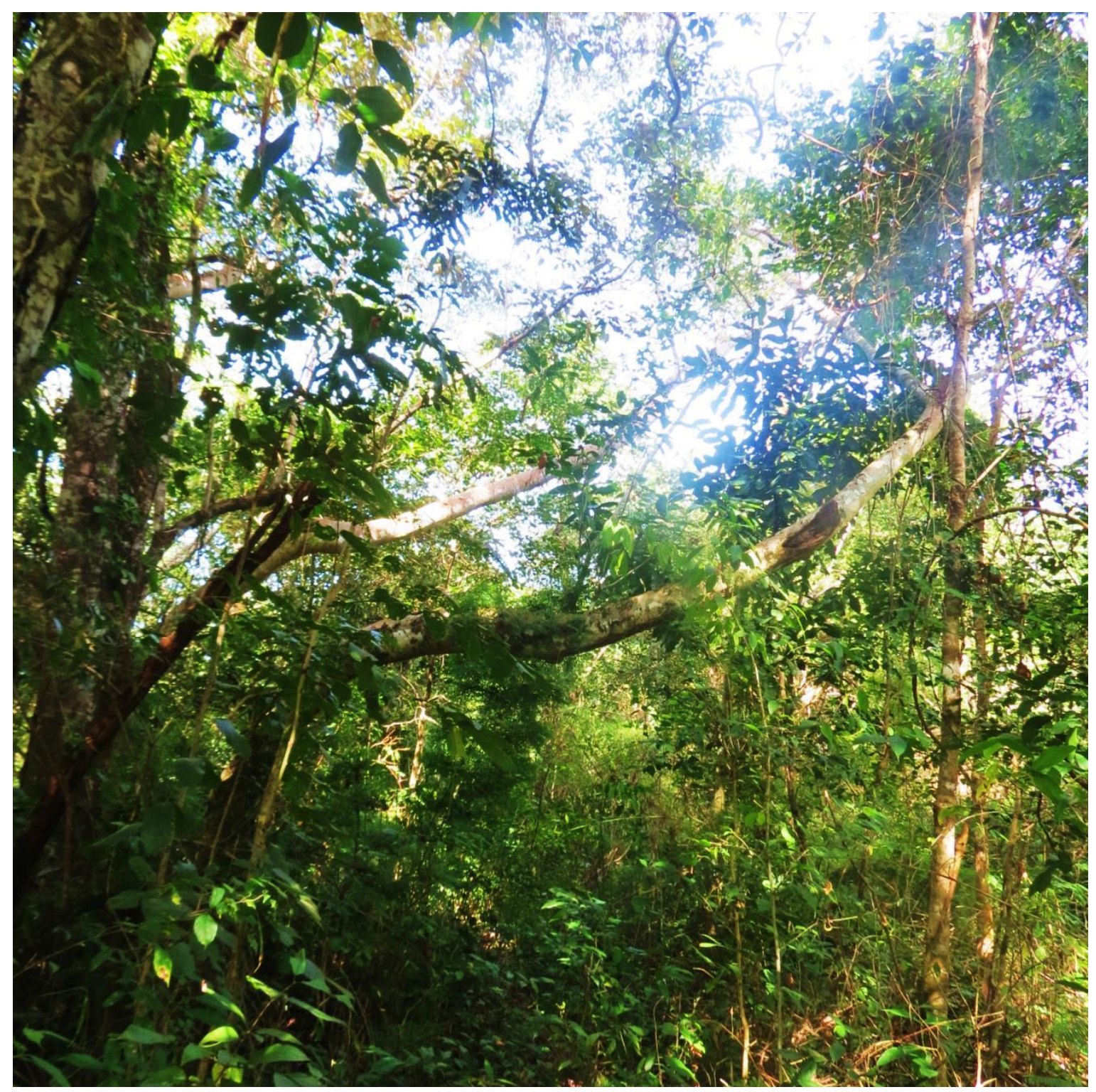

Figura 2 - Visão geral da vegetação encontrada na Área 1 (Fonte: Arquivo Pessoal). 


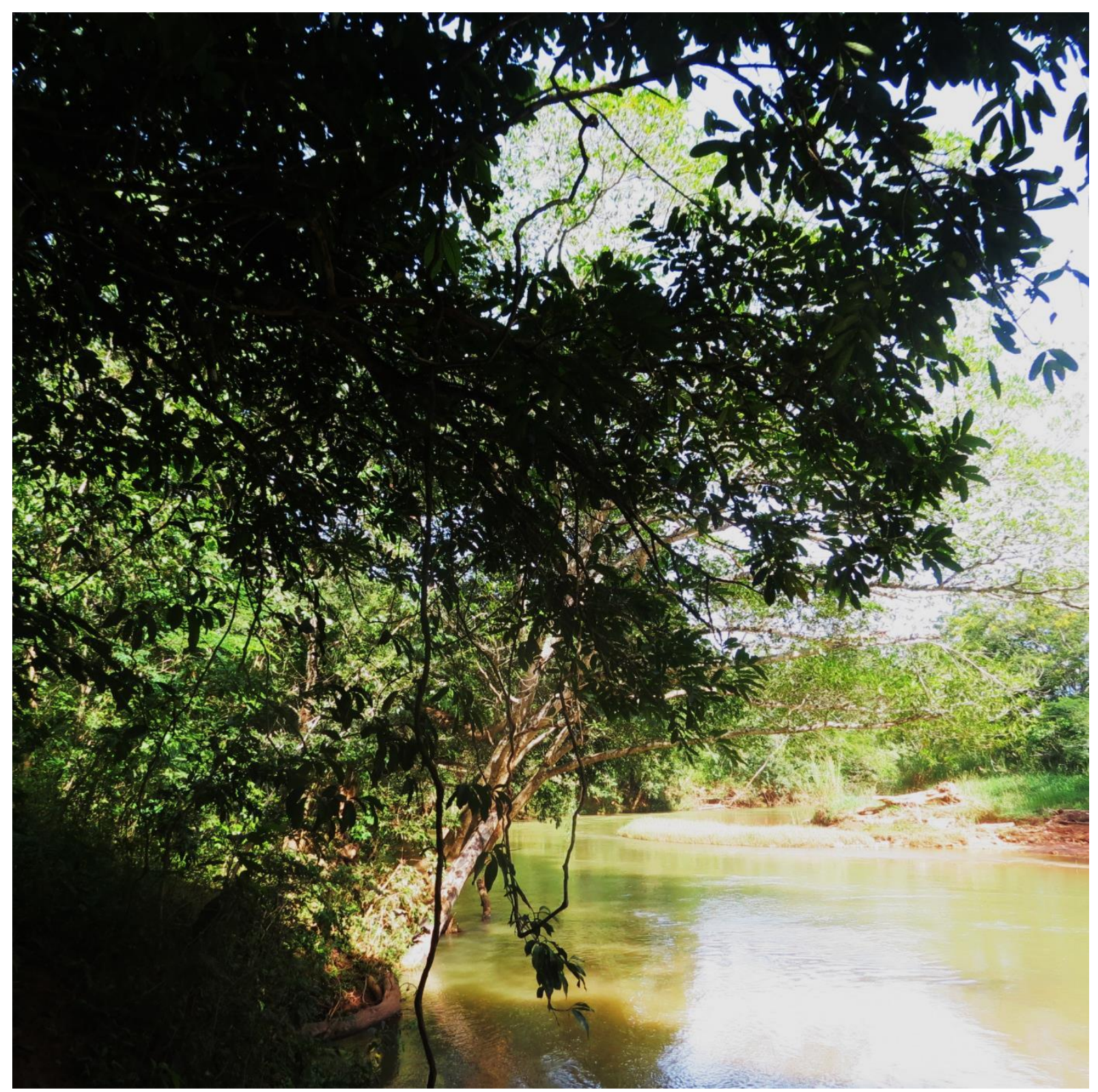

Figura 3 - Visão geral do trecho do Rio Maranhão encontrado na Área 1 (Fonte: Arquivo Pessoal). 


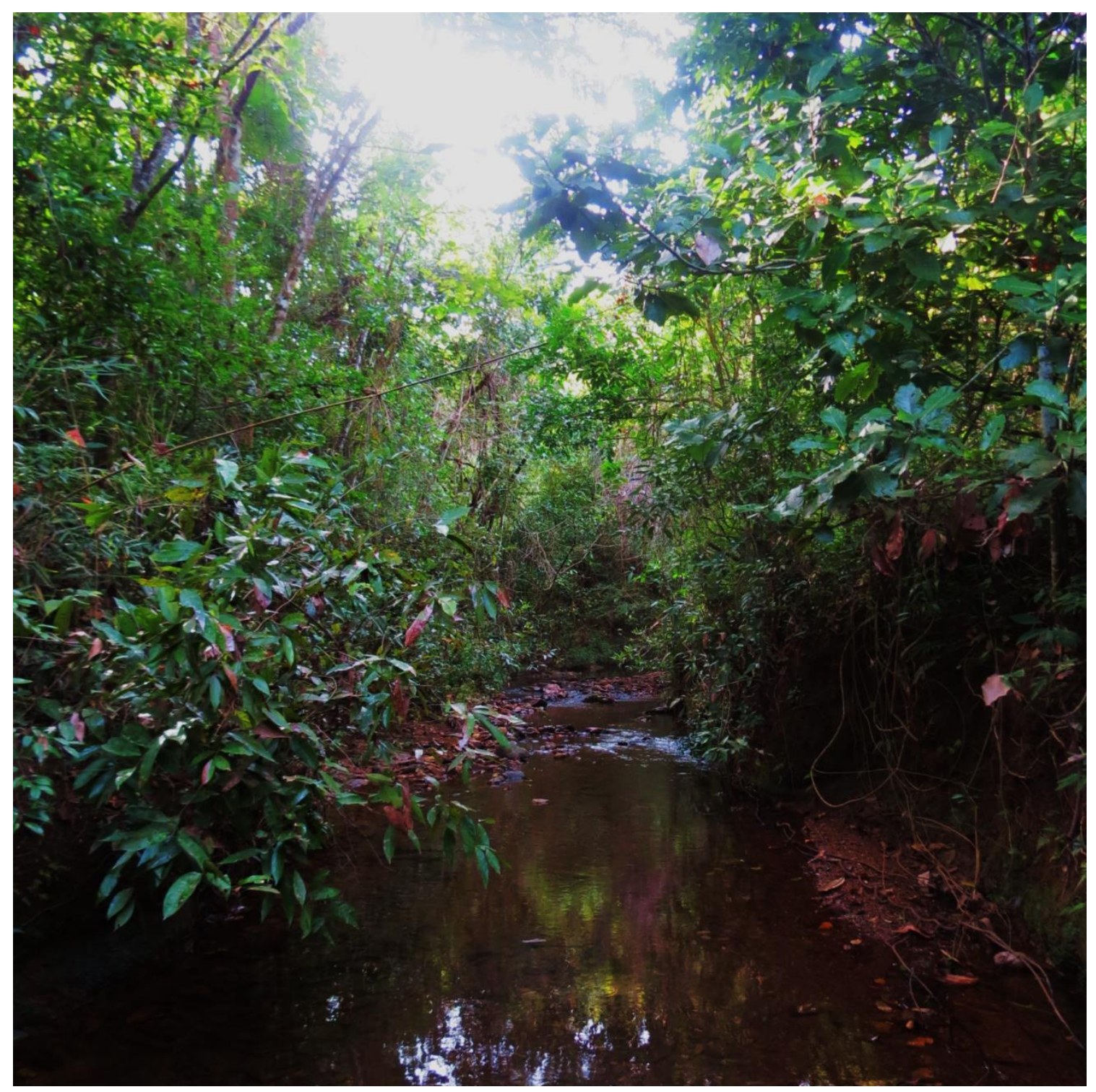

Figura 4 - Visão geral da vegetação e do afluente do Rio Maranhão encontrados na Área 2 (Fonte: Arquivo Pessoal). 


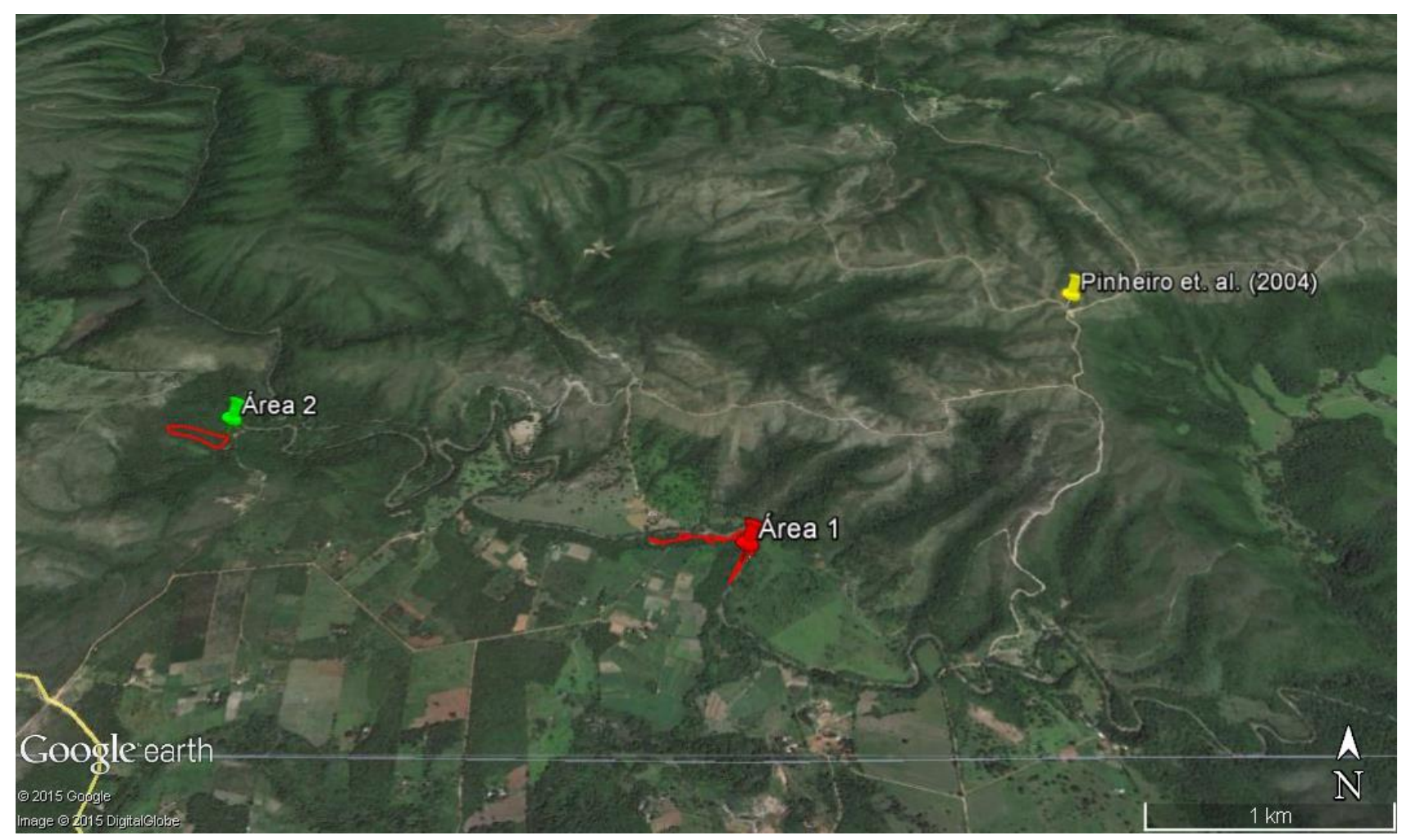

Figura 5 - Mapa com as localizações das áreas de estudo (Fonte: Google Earth Pro).

\section{Métodos de campo}

Foram realizadas uma ou duas viagens a campo por semana, com duração entre uma e quatro horas de permanência nas áreas de estudo, durante os meses de Agosto e Setembro de 2014 (estação seca = ES) e de Janeiro a Maio de 2015 (estação chuvosa = EC), totalizando 102 horas de observação (51 horas/observador).

As aves foram localizadas através de sua vocalização, observação dos poleiros que costumam frequentar, ou ainda, através da realização de playbacks utilizando-se, para isto, um celular do modelo Motorola Moto G. Para auxiliar a observação e identificação dos insetos atacados e consumidos foram utilizados uma câmera fotográfica Canon PC 1680 e dois binóculos (Wide-anjo Seeker 10 X 50 w e KarlZeiss Jena 8 × 30 w). 
Os indivíduos encontrados no campo foram identificados quanto ao sexo (facilmente reconhecidos pelas manchas branca ou parda no pescoço, Figura 1) e observados quanto ao seu comportamento alimentar enquanto permaneceram no campo de visão dos observadores. Informações como o horário e o local da captura de presas (no ar, sobre a vegetação ou no chão), bem como as reações das aves durante a captura da presa, seu manuseio (subjugação) e após o consumo, também foram registradas.

Com exceção de presas muito pequenas $(<1 \mathrm{~cm})$, tratadas em conjunto como "Miscelânea", e geralmente capturadas no ar, incluindo muitos micro-Hymenoptera e Diptera, a grande maioria das presas capturadas e consumidas por esta ave foi identificada ao nível de ordem.

\section{Aves investigadas no campo}

Durante a estação seca, a maioria das observações na Área 1 foi obtida a partir de um casal de aves que permaneceu ao redor dos mesmos poleiros durante longos períodos de tempo. Também foram feitas observações de um segundo casal, e observações ocasionais de indivíduos solitários.

Um casal na fase inicial de formação (ambos os sexos forrageando por conta própria) foi também encontrado e observado na Área 2, além de vários indivíduos isolados. Em ambos os casos foi possível permanecer a pouco mais de $3 \mathrm{~m}$ das aves durante boa parte das observações sem, aparentemente, causar prejuízo ou alterações no comportamento destas.

Ao contrário da estação seca, a maioria das observações obtidas na estação chuvosa foram de indivíduos adultos isolados que, além de permaneceram pouco tempo nos poleiros, foram mais dificilmente localizados e observados, devido ao crescimento da vegetação. 


\section{Análise de variações na dieta e testes estatísticos empregados}

Para obter uma análise mais objetiva nos estudos comparativos sobre a dieta da $G$. ruficauda foram definidos três grandes grupos de insetos com marcantes variações em seu tamanho, abundância relativa e local de captura em que a presa foi capturada, da seguinte forma: (1) insetos pequenos, coletivamente denominados "Miscelânea", provavelmente incluindo muitos micro-Hymenoptera e Diptera dificilmente identificados a distancia e capturados em voo; (2) "insetos intermediários", constituídos exclusivamente por vespas e formigas (Hymenoptera); e (3) "insetos grandes", incluindo Lepidoptera, Odonata, Orthoptera, Coleoptera e Mantodea e proporcionalmente menos numerosos na dieta da G. ruficauda no Brasil central. A proporção de insetos nestes três grupos foi posteriormente comparada através de Tabelas de Contingência (Teste G) entre sexos, locais (Área 1 x Área 2) e estações do ano (Estação seca $\times$ Estação chuvosa). Este teste foi também utilizado nas comparações envolvendo os resultados deste estudo com os estudos de Chai (1986) na América central e de Pinheiro et al.(2004), em área próxima às áreas de estudo desta dissertação (Figura 5). Para a realização destes testes foi empregado o pacote estatístico BioEstat versão 5.3.

\section{RESULTADOS}

No total foram observados 717 itens alimentares atacados e consumidos por machos e fêmeas de G. ruficauda nas duas estações e áreas de estudo (Tabela 1). Miscelânea foi o grupo mais consumido por ambos os sexos, seguidos de Hymenoptera, Diptera, Lepidoptera, Odonata e três outras ordens representadas por apenas um indivíduo: Coleoptera, Orthoptera e Mantodea (Figura 6 A, B). Sete indivíduos não foram identificados quanto a sua ordem, seja pelo fato do ataque ter sido muito rápido ou por haver alguma obstrução impedindo a visão.

A maioria dos Hymenoptera observados na dieta da $G$. ruficauda foi constituída por vespas, mas algumas formigas (Formicidae), um item ainda não observado em outros estudos sobre a dieta desta ave, também foram observadas. 
Depois de capturadas, muitas vespas foram repetidamente batidas contra o poleiro de alimentação, aparentemente para a remoção de seus ferrões antes de seu consumo, um comportamento também observado por Pinheiro et al. (2004) e por Antas (2009). Este comportamento também foi observado em relação a alguns Lepidoptera grandes (especialmente alguns Pieridae) que tiveram suas asas removidas antes de consumi-los. No caso de algumas borboletas impalatáveis, como Heliconius sp. (Nymphalidae), foi também observado que, após a sua captura algumas aves podem arremessá-las para cima e pega-las novamente, repetindo esse comportamento uma ou duas vezes antes de liberta-las definitivamente. De acordo com Pinheiro \& Campos (2013) este comportamento poderia ajudar as aves na verificação de sua palatabilidade.

Muitos Diptera identificados neste estudo foram capturados sobre a carcaça de um canídeo em avançado estado de decomposição, próximo a um dos poleiros mais utilizados pelo casal de aves encontrado na Área 2 durante a estação seca.

O consumo de Miscelânea permaneceu relativamente semelhante em ambas as estações (Figura 6). Durante a estação seca houve um maior consumo de Hymenoptera e Diptera, em relação à estação chuvosa, bem como a única predação de um Coleoptera. Já na estação chuvosa houve um aumento de Odonata e Lepidoptera e a predação de Orthoptera e Mantodea. 
Tabela 1 - As ordens de insetos e o total de indivíduos capturados pelo Galbula ruficauda neste e outros estudos realizados no Brasil central e na Costa Rica. Dados relativos a sexo e estações do ano deste estudo foram agrupados.

$\begin{array}{ccccc}\begin{array}{c}\text { Local de } \\ \text { estudos }\end{array} & \begin{array}{c}\text { Brasil central } \\ \text { (Área 1) }\end{array} & \begin{array}{c}\text { Brasil central } \\ \text { (Área 2) }\end{array} & \begin{array}{c}\text { Brasil central } \\ \text { (Pinheiro et al. 2004) }\end{array} & \begin{array}{c}\text { Costa Rica } \\ \text { Chai (1986) }\end{array} \\ \begin{array}{c}\text { Estações } \\ \text { estudadas }\end{array} & \text { ES + EC } & \text { ES + EC } & \text { EC } & \text { EC } \\ \begin{array}{c}\text { Tempo de } \\ \text { observação } \\ \text { no campo }\end{array} & 51 \mathrm{hs} & 51 \mathrm{hs} & 38 \mathrm{hs} & 26 \mathrm{hs} \\ \end{array}$

\begin{tabular}{ccccc} 
Miscelânea & $218(63 \%)$ & $200(53 \%)$ & $124(39 \%)$ & $236(77 \%)$ \\
Hymenoptera & $74(22 \%)$ & $120(32 \%)$ & $101(32 \%)$ & $15(5 \%)$ \\
Diptera & $30(9 \%)$ & $11(3 \%)$ & $5(2 \%)$ & $12(4 \%)$ \\
Lepidoptera & $15(4 \%)$ & $25(7 \%)$ & $47(15 \%)$ & $15(5 \%)$ \\
Odonata & $4(1 \%)$ & $10(3 \%)$ & $8(3 \%)$ & $18(6 \%)$ \\
Coleoptera & $0(0 \%)$ & $1(0 \%)$ & $0(0 \%)$ & $0(0 \%)$ \\
Orthoptera & $0(0 \%)$ & $1(0 \%)$ & $19(6 \%)$ & $0(0 \%)$ \\
$\begin{array}{c}\text { Mantodea } \\
\text { Hemiptera } \\
\text { (cigarras) } \\
\text { Não }\end{array}$ & $0(0 \%)$ & $1(0 \%)$ & $0(0 \%)$ & $0(0 \%)$ \\
identificado & $2(1 \%)$ & $0(0 \%)$ & $8(3 \%)$ & $10(3 \%)$ \\
Total & 343 & $5(1 \%)$ & - & - \\
\hline
\end{tabular}




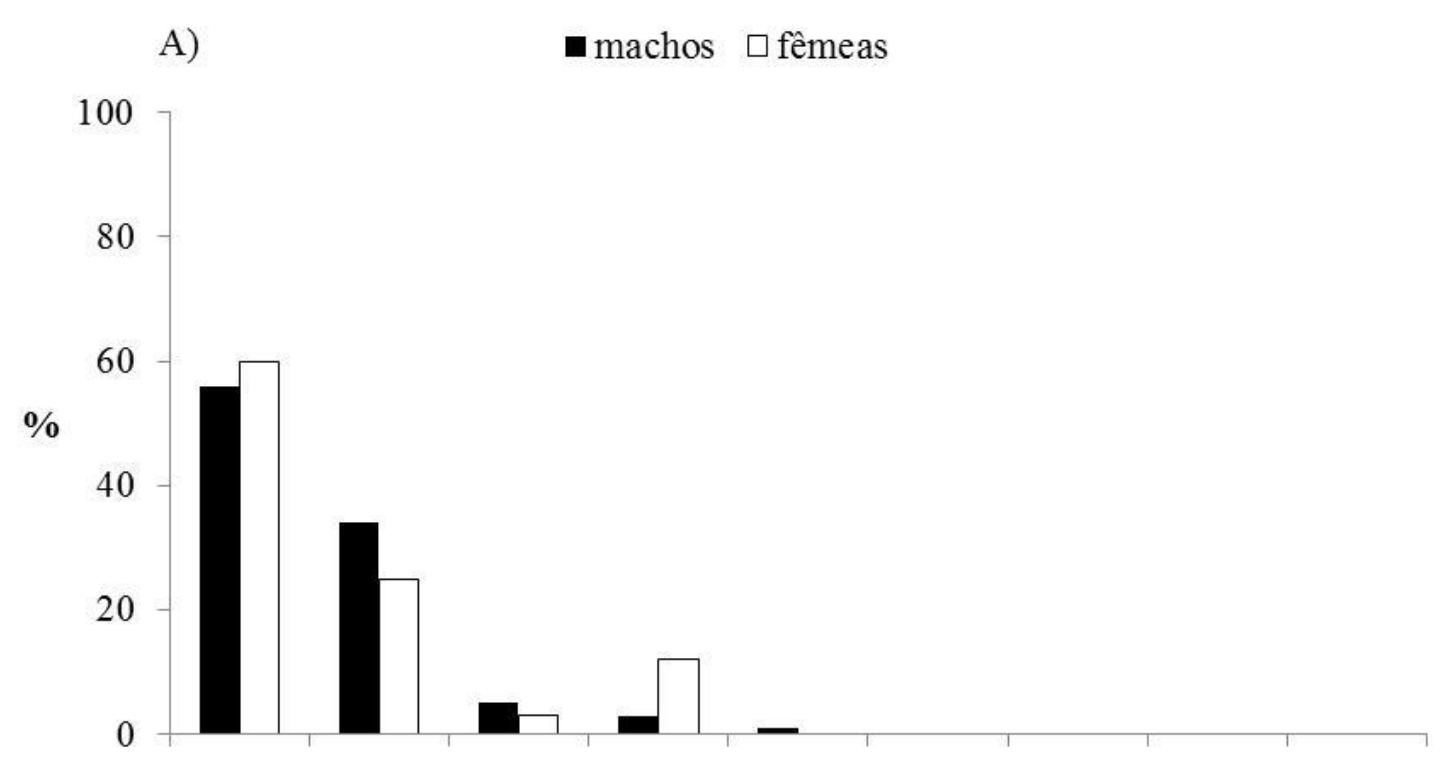

B)

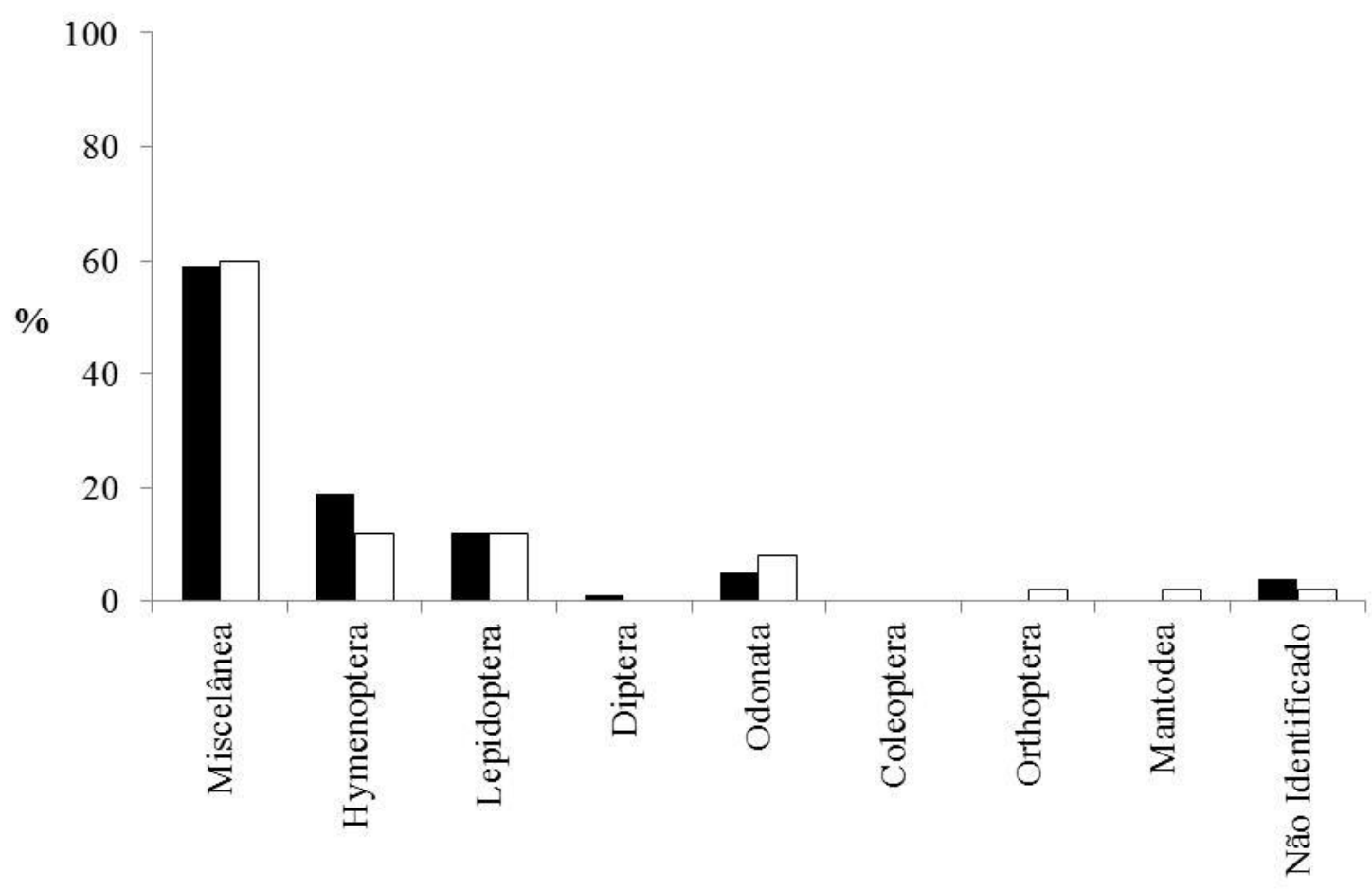

Figura 6 - Proporção (\%) de diferentes ordens de insetos na dieta de machos e fêmeas de Galbula ruficauda nas estações seca (Figura 6. A; $n=570$ itens alimentares) e chuvosa (Figura 6. B; $n=147$ itens alimentares). Áreas de estudo (1 e 2) foram agrupadas. 
Todos os Miscelânea (100\%), Diptera (100\%) e a maioria de Hymenoptera (88\%) foram capturados no ar e, em alguns casos, sem a necessidade da ave sair do poleiro para capturá-los. Todos os Coleoptera, Orthoptera e Mantodea (100\%) e boa parte das Lepidoptera $(55,56 \%)$, Odonata $(31 \%)$ foram capturados quando pousados sobre a vegetação (Figura 7). Foi observado que as aves acompanharam, com a visão, a trajetória de indivíduos pertencentes à varias ordens e esperaram até que pousassem antes de ataca-los.

As proporções de insetos pequenos, intermediários e grandes na dieta da $G$. ruficauda, incluindo os dados obtidos por Pinheiro et al. (2004) e Chai (1986), estão representadas na Figura 8, separados por sexo, estação do ano e área de estudos. O grupo "Insetos pequenos" foi o mais consumido por ambos os sexos nas duas áreas e estações do ano (Figura 8 A-D), um fenômeno também observado no estudo de Pinheiro et al. (2004; Figura 8 E) e de Chai (1986) na Costa Rica (Figura 8 F). "Insetos Intermediários" foi o segundo grupo mais consumido em ambos os estudos realizados em áreas de cerrado no Brasil central, mas não na Costa Rica, onde foi encontrada uma maior proporção de insetos grandes quando comparados aos resultados do Brasil central.

Testes estatísticos envolvendo as comparações das proporções de itens pequenos, intermediários e grandes na dieta do G. ruficauda entre sexos, estações do ano e áreas de estudo são apresentados na Tabela 2. Não foram encontradas diferenças significativas entre sexos em nenhum dos testes realizados (testes $\mathrm{n}^{0} \mathbf{1}$ 4). Por outro lado, foram encontradas diferenças significativas em todos os testes envolvendo as estações do ano (testes ํㅜ 5-8), mostrando claramente como a dieta do $G$. ruficauda se modifica nestes dois períodos. Com exceção de dois testes (testes no 9 e 13) não foram encontradas diferenças significativas em nenhum dos testes envolvendo comparações entre áreas de estudo no Brasil central (testes $\mathrm{n}^{\circ}$ $10,11,12,14,15,16)$. Entretanto, diferenças altamente significativas foram encontradas entre as áreas investigadas neste estudo e os resultados obtidos por Chai (1986) na América Central. 


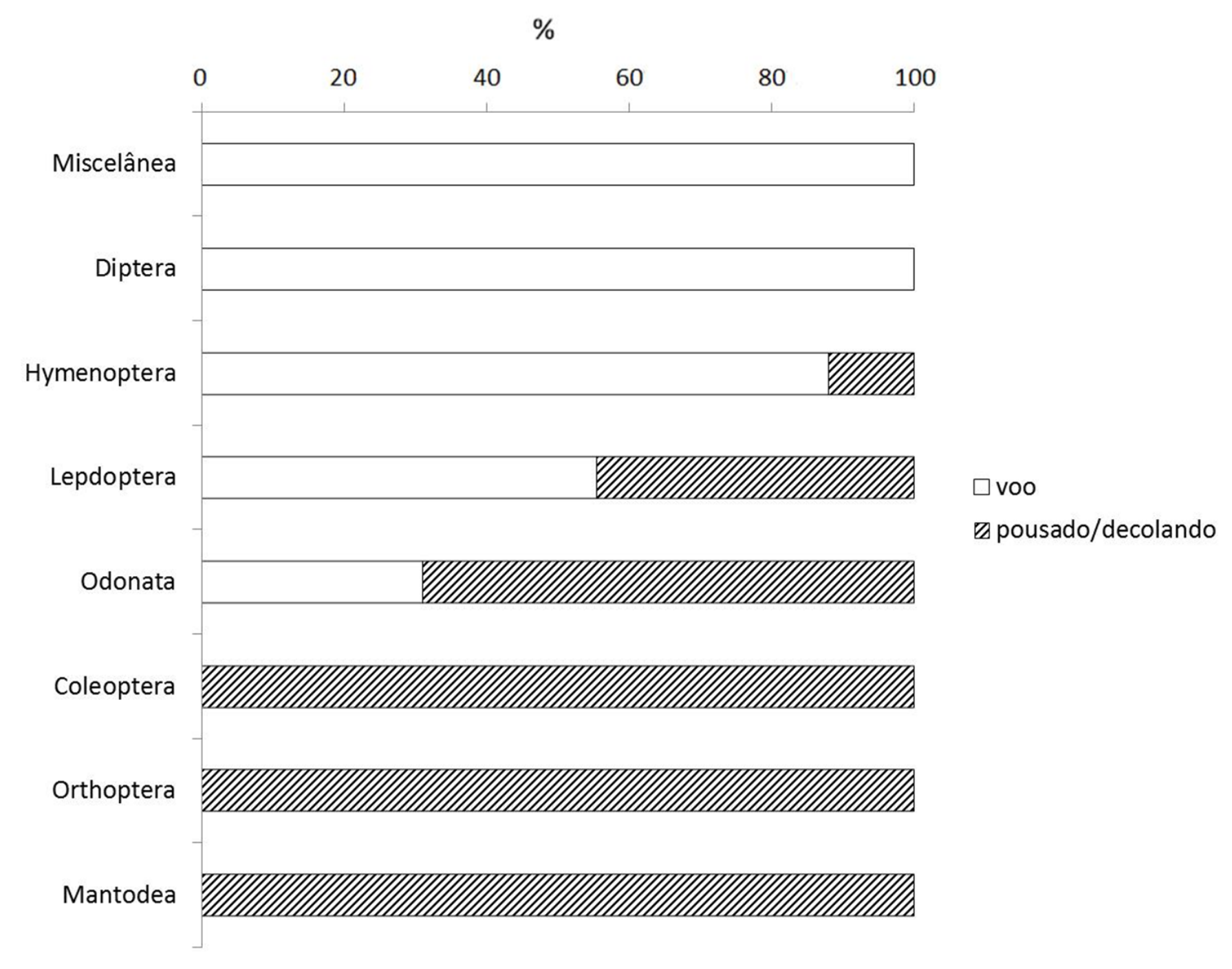

Figura 7 - Proporção (\%) de indivíduos capturados no substrato (ou decolando) e em voo em cada uma das ordens de insetos consumidas pelo Galbula ruficauda. 


\section{Área 1}

A)

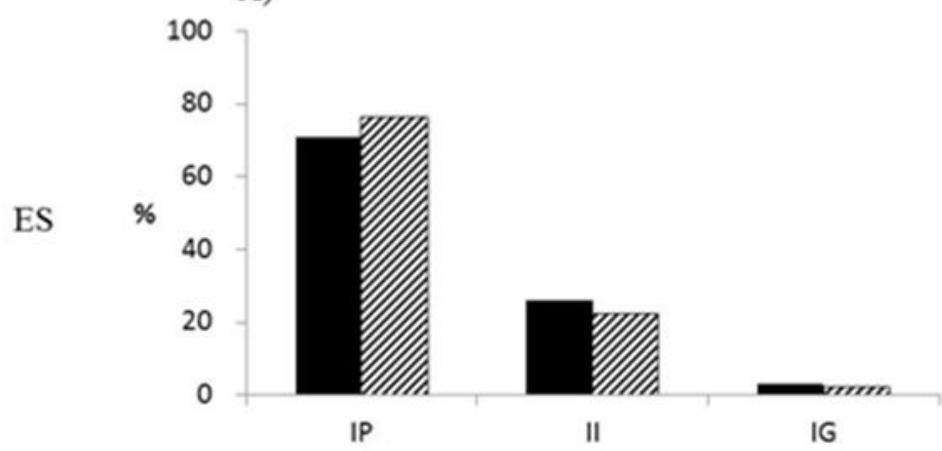

C)

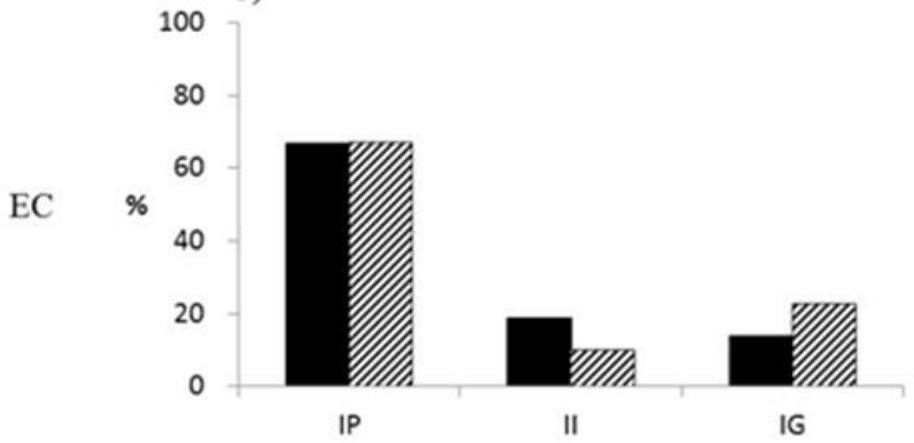

E)

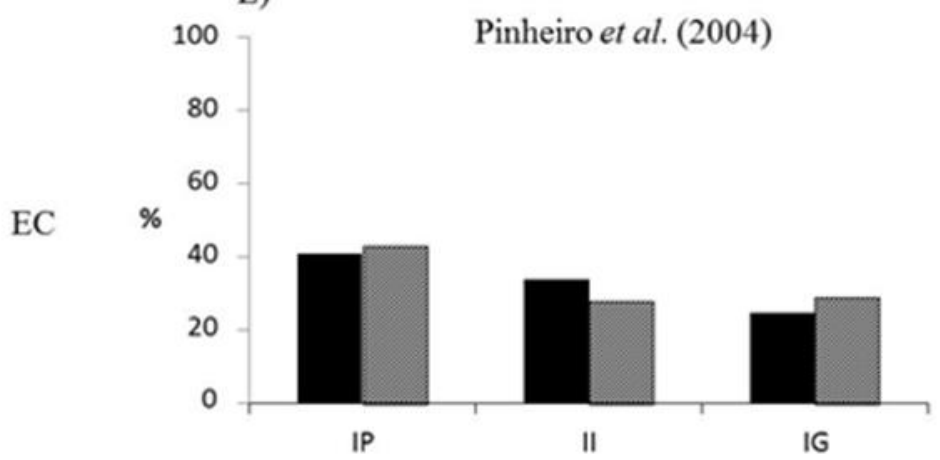

Área 2

B)

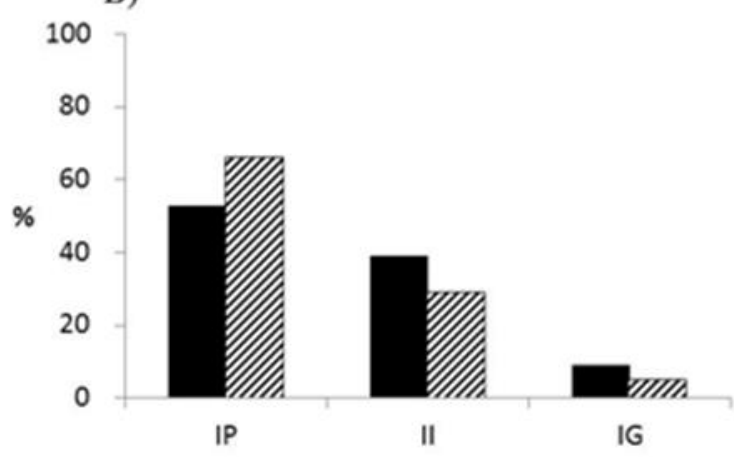

D)

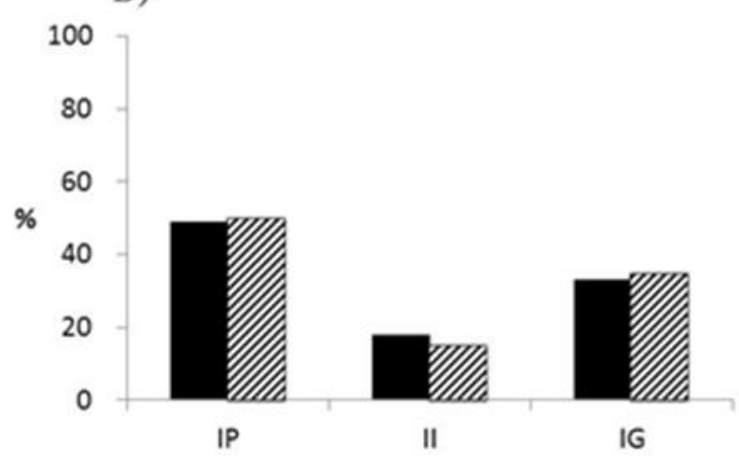

F)

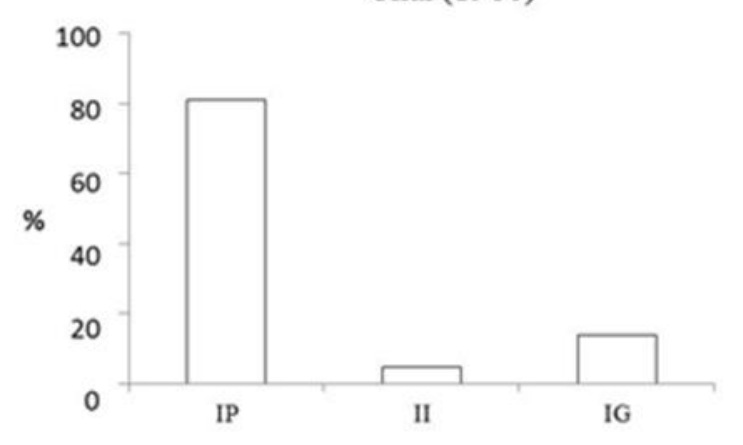

Figura 8 - Proporção (\%) de insetos pequenos (Miscelânea), insetos intermediários (Hymenoptera) e insetos grandes (Lepidoptera, Odonata, Coleoptera, Orthoptera, Mantodea e não identificados) na dieta do Galbula ruficauda separados por sexo, áreas de estudo e estações do ano. 
Tabela 2 - Resultados dos testes G (com correção de Williams) nas comparações realizadas entre sexo, estações do ano (Estação seca e Estação chuvosa) e áreas de estudo. Todos os testes com 2 graus de liberdade.

\begin{tabular}{|c|c|c|c|c|}
\hline & & COMPARAÇÕES & G (Williams) & $P$ \\
\hline \multirow{4}{*}{ ENTRE SEXOS } & 1 & $\widehat{\partial}{ }^{\lambda}$ (Área 1; ES; $\left.n=104\right) x+q$ (Área 1; ES; $n=151$ ) & 0,814 & 0,666 \\
\hline & 2 & 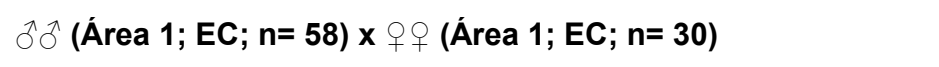 & 2,007 & 0,367 \\
\hline & 3 & 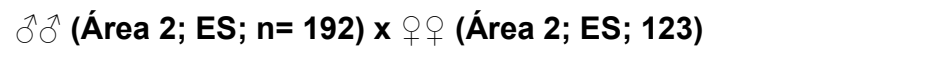 & 5,764 & 0,056 \\
\hline & 4 & 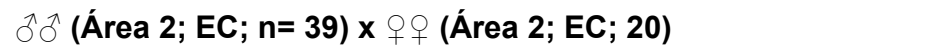 & 0,081 & 0,961 \\
\hline \multirow{4}{*}{ ENTRE ESTAÇÕES } & 5 & 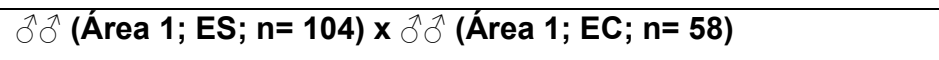 & 6,858 & 0,032 \\
\hline & 6 & 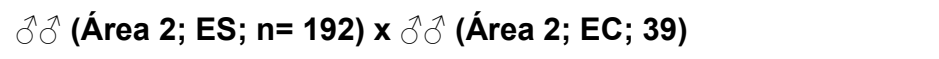 & 15,795 & 0,0004 \\
\hline & 7 & 许 (Área 1; ES; n=151 ) x $q$ (Área 1; EC; 30) & 15,399 & 0,0005 \\
\hline & 8 & 우 (Área 2; ES; n=123 ) x & 12,795 & 0,0017 \\
\hline \multirow{10}{*}{ ENTRE ÁREAS } & 9 & 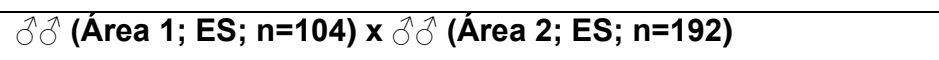 & 10,983 & 0,0041 \\
\hline & 10 & 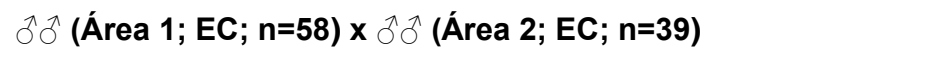 & 5,241 & 0,073 \\
\hline & 11 & $q+$ (Área 1; ES; n= 151) x $q$ (Área 2; ES; n=123) & 4,080 & 0,13 \\
\hline & 12 & 우 (Área 1; EC; n=30) x + (Área 2; EC; n= 20) & 1,298 & 0,523 \\
\hline & 13 & 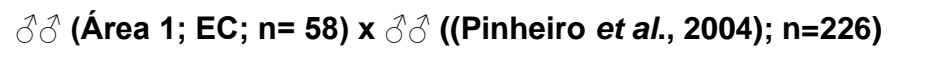 & 11,744 & 0,0028 \\
\hline & 14 & 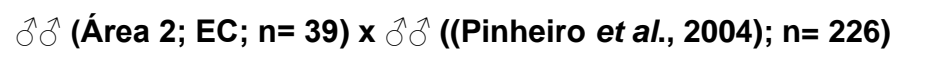 & 4,488 & 0,106 \\
\hline & 15 & ㅇ (Área 1; EC; $\mathbf{n = 3 0 )} \mathbf{x}$ 우 ((Pinheiro et al., 2004); $\mathbf{n = 8 6}$ ) & 5,775 & 0,0557 \\
\hline & 16 & 우 (Área 2; EC; $\mathbf{n = 2 0 ) ~ x ~}+$ ((Pinheiro et al., 2004) ; n= 86) & 1,5332 & 0,46 \\
\hline & 17 & 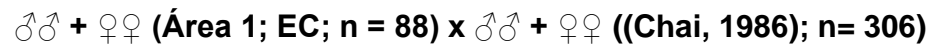 & 11,334 & 0,004 \\
\hline & 18 & ồ + $+q$ (Área 2; EC; $\mathbf{n}=\mathbf{5 9}) \times$ x & 24,080 & $<0.0001$ \\
\hline
\end{tabular}




\section{DISCUSSÃO}

Apesar de ser conhecido por muitos autores como um predador especializado em Lepidoptera (Chai 1986, Mallet \& Barton 1989, Tobias et al. 2002; Langhan 2004, 2006) os dados obtidos no presente estudo bem como os de Chai (1986) e Pinheiro et. al (2004) revelam que o principal item da dieta do G. ruficauda são pequenos insetos alados capturados no ar, os quais foram consumidos em quantidade superior aos demais, por ambos os sexos, em ambas as áreas e em ambas as estações. Segundo Chai (1986) esse grupo de insetos corresponde a $40 \%$ da biomassa consumida na Costa Rica, sendo possível que um número semelhante seja encontrado no Brasil central.

Além disto, podemos observar que os Hymenoptera constituem o segundo grupo de insetos mais consumido pela $G$. ruficauda em todos os resultados obtidos no Brasil central, correspondendo a $27 \%$ do consumo total, no presente estudo (sexos, áreas, e estações do ano agrupadas) e 32\% no estudo de Pinheiro et al. (2004). Estes resultados também corroboram o estudo de Fry (1970a) realizado no Mato Grosso que também encontrou um elevado numero de Hymenoptera no conteúdo estomacal desta ave.

Além de miscelânea e das sete ordens encontradas neste estudo, outros autores também encontraram alguns Hemiptera (Pinheiro et al. 2004; Lopes et al. 2005) e Phasmida (Lopes et al. 2005) na dieta desta ave. É possível que estas diferenças ocorram devido a diferenças na disponibilidade destas ordens nos diversos locais amostrados.

As comparações envolvendo a presença de insetos pequenos, intermediários e grandes entre sexos não mostraram diferenças significativas, corroborando os dados de Pinheiro et al. (2004). Essa semelhança já poderia ser esperada, uma vez que os sexos possuem tamanhos semelhantes, vivem nos mesmos habitats e microhabitats, e apresentam os mesmos comportamentos em relação aos vários tipos de presas consumidos por eles. 
Já nas comparações realizadas entre as estações seca e chuvosa foram encontradas diferenças marcantes. Estas diferenças poderiam ser esperadas em função das fortes variações climáticas que ocorrem nestas estações, especialmente em relação à temperatura e a pluviosidade que afetam a estrutura da vegetação e a abundancia de insetos utilizados como alimento por esta ave (Marinoni \& Ganho 2003; Torres \& Madi-Ravazzi 2006).

A proporção de insetos pequenos, intermediários e grandes na dieta da $G$. ruficauda não tende a diferir significativamente nas áreas de estudo localizadas no Brasil central (Áreas 1 e 2 e a área utilizada no estudo de Pinheiro et al. 2004). As exceções encontradas (apenas nos testes $n^{\circ} 9$ e 13) envolveram machos da Área 1 e podem ser resultado de diferenças na estrutura da vegetação desta área (uma área de mata de galeria a beira do Rio Maranhão, bem maior que as matas de galeria dos pequenos córregos afluentes deste rio, como a Área 2 e a área estudada por Pinheiro et al. (2004)). Em pelo menos um teste realizado com fêmeas nesta mesma área (teste 15) o resultado obtido foi muito próximo ao nível de significância de 0.05. Todavia, é possível que outros fatores, não quantificados ou não observados, também tenham afetado estes resultados.

Já as comparações das Áreas 1 e 2 com os dados de Chai (1986) revelaram uma grande diferença na proporção destes três grupos de insetos entre estes locais e, conforme citado acima, poderiam ser devidas à maior abundancia de vespas (Hymenoptera) no Brasil central, que por sua vez, poderia levar a uma diminuição no consumo de insetos grandes quando comparados aos dados da Costa Rica. Alguns estudos realizados no cerrado tem mostrado uma alta densidade de vespas nesta região (Henriques et al. 1992; Henriques \& Palma 2015). Entretanto, não existem dados disponíveis sobre a abundância de vespas nas florestas da Costa Rica que pudessem corroborar esta hipótese.

\section{CONCLUSÕES}

1. Insetos pequenos capturados no ar são os principais itens da dieta do G. ruficauda tanto no Brasil central quanto na Costa Rica; 
2. Vespas (Hymenoptera) são o segundo item mais consumido no Brasil central e provavelmente em todo o Bioma Cerrado.

3. A proporção de insetos pequenos, intermediários e grandes na dieta do G. ruficauda não difere significativamente entre sexos, mas difere entre estações do ano;

4. A proporção de insetos pequenos, intermediários e grandes na dieta do G. ruficauda pode diferir ou não entre localidades relativamente próximas entre si no Brasil central.

5. A proporção de insetos pequenos, intermediários e grandes na dieta do G. ruficauda no Brasil central difere significativamente dos dados obtidos na Costa Rica. 


\section{REFERÊNCIAS BIBLIOGRÁFICAS}

ANTAS, P. T. Z . 2009. Pantanal: Guia de Aves - Espécies da Reserva Particular do Patrimônio Natural do SESC - Pantanal disponível em: http://www.avespantanal.com.br/paginas/index.htm (acesso em 5/2/2013).

BERLA, H. F. 1944. Lista das aves colecionadas em Pedra Branca, município de Paratí, Estado do Rio de Janeiro, com algumas notas sobre sua biologia. Boletim do Museu Nacional, 18, 1-21.

BROWER, L. P. 1984. Chemical defence in butterflies. In: Vane-Wright, R. I. \& Ackery, P. R. (eds.). The biology of butterflies. Academic Press, .pp. 109-134.

CHAI, P. 1986. Field observations and feeding experiments on the responses of rufous-tailed jacamars (Galbula ruficauda) to free-flying butterflies in a tropical rainforest. Biological Journal of the Linnean Society, 29, 161-189.

FRY, C. H. 1970a. Convergence between jacamars and bee-eaters. Ibis, 112(2), 257- 259.

FRY, C. H. 1970b. Ecological distribution of birds in north-eastern Mato Grosso State, Brazil. Anais da Academia Brasileira de Ciências, 42(2), 275.

GOODLAND, R. 1971. A physiognomic analysis of the Cerrado vegetation of Central Brasil. The Journal of Ecology, 59, 411- 419.

GWYNNE, J. A., RIDGELY R. S., TUDOR G., \& ARGEL M. 2010. Aves do Brasil: Pantanal \& Cerrado. São Paulo: Wildlife Conservation Society, Ed. Horizonte.

HENRIQUES, R. P. B., DINIZ ROCHA, I. R., \& KITAYAMA, K. 1992. Nest density of some social wasp species in Cerrado Vegetation of Central Brazil (Hymenoptera: Vespidae). Entomologia Generalis, 17(4), 267-268.

HENRIQUES, R. P. B., \& PALMA, A. R. T. 2015. Bird predation on nest of a social wasp in Brazilian cerrado. International Journal of Tropical Biology and Conservation, 46(4), 1145-1146. 
KERNAN, C. \& FOWLER, N. 1995. Differential substrate use by epiphytes in Corcovado National Park, Costa Rica: a source of guild structure. Journal of Ecology, 83, 65-73.

LANGHAM, G. M. 2004. Specialized avian predators repeatedly attack novel color morphs. Evolution, 58, 2783-2787.

LANGHAM, G. M. 2006. Rufous-tailed jacamars and aposematic butterflies: do older birds attack novel prey? Behavioral Ecology, 17(2), 285-290.

MARINONI, R. C., \& GANHO, N. G. 2003. Seasonality of Nyssodrysina lignaria (Bates) (Coleoptera, Cerambycidae, Lamiinae), in the Paraná State, Brazil. Revista Brasileira de Zoologia, 20(1), 141-152.

MELO-JÚNIOR, T. A. 2001. Comportamento alimentar do cuitelão (Jacamaralcyon tridactyla) em duas reservas no Estado de Minas Gerais. In: Straube, F. C. (Ed.). Ornitologia sem Fronteiras, Boticário, Curitiba. Pp: 277-278.

MOOJEN, J., CARVALHO, J., \& LOPES, H. 1941. Observações sobre o conteúdo gástrico das aves brasileiras. Memórias do Instituto Oswaldo Cruz, 36(3), 405-444.

NOVAES, F. C., \& LIMA, M. D. F. C. 1998. Aves da grande Belém: Municípios de Belém e Ananindeua, Pará. PR/McT/Cnpq.

PINHEIRO C. E. G, BAGNO, M. A. \& BRANDÃO, R. A. 2004. Diet and foraging behavior of the rufous-tailed jacamar (Galbula ruficauda, Galbulidae) in central Brazil. Ararajuba, 11, 241-243.

PINHEIRO, C. E. G. 2011. On the evolution of warning coloration, Batesian and Mullerian mimicry in Neotropical butterflies: the role of jacamars (Galbulidae) and tyrant-flycatchers (Tyrannidae). Journal of Avian Biology, 42, 277-281.

PINHEIRO, C. E. G. \& CAMPOS, V. C. 2013. Do rufous-tailed jacamars (Galbula ruficauda) play with aposematic butterflies? Ornitología Neotropical, 24, 365367 , 
POULIN, B.; LEFEBVRE, G. \& MCNEIL, R. 1994. Diets of land birds from northeastern Venezuela. Condor, 96, 354-361.

SCHUBART, O., AGUIRRE, A. C., \& SICK, H. 1965. Contribuição para o conhecimento da alimentação das aves brasileiras. Arquivos de Zoologia, 12, 95-249.

SCOTT, S. L. 2013. Feeding ecology of birds in a mist belt forest in South Africa. Dissertação de mestrado. Universidade de Witeatersrand, Johannesburg, África do Sul.

SHERRY, T. W. 1983. Galbula ruficauda. Costa Rican Natural History. University of Chicago Press, Chicago, pp. 579-581.

SICK, H. 1993. Birds in Brazil. Princeton Univ. Press, Princeton, New Jersey.

SICK, H.1997. Ornitologia Brasileira. Rio de Janeiro. Nova Fronteira.

SKUTCH, A. F. 1937. Life-history of the Black-chinned Jacamar. Auk, 54(2), 135146.

SKUTCH, A. F. 1963. Life history of the Rufous-tailed jacamar Galbula ruficauda in Costa Rica. Ibis, 105(3), 354-368.

SLUD, P. 1964. The birds of Costa Rica: distribution and ecology. Bulletin of the American Museum of Natural History, 128, 1-430.

TERBORGH, J.; ROBINSON, S. K.; PARKER, T. A.; MUNN, C. A., \& PIERPONT, N. 1990. Structure and organization of an Amazonian forest bird community. Ecological Monographs, 60(2), 213-238.

TOBIAS J. A, ZÜCHNER, T. \& DE MELO-JÚNIOR T. A. 2002. Family Galbulidae (Jacamars). In Handbook of the birds of the world del Hoyo J, Elliott A, Saragatal J., 7,pp. 74-101. Eds. Barcelona, Spain:Lynx edicions

TORRES, F. R., \& MADI-RAVAZZI, L. 2006. Seasonal variation in natural populations of Drosophila spp. (Diptera) in two woodlands in the State of São Paulo, Brazil. Iheringia, 96(4), 437-444 
VASCONCELOS, M. F, HOFFMANN, D., ARAÚJO, M. C., \& VASCONCELOS, P. N. 2015. Bird-termite interactions in Brazil: A review with perspectives for future studies. Biota Neotropica, 15(1), 1-22. 